\title{
The minimal interaction induced by the translation subgroup has a gap in the low-symmetric state.
}

\author{
Alexander Braginsky ${ }^{1}$
}

${ }^{1}$ YUFU

February 21, 2022

\begin{abstract}
The paper investigates the low-symmetric state of the compensating field of the distortion tensor and proves that there is a gap in this state. It is shown that the distortion tensor is the compensating field of the minimal interaction induced by a translation subgroup. In this paper, for the first time, an exact wave solution for sound pressure in a continuous medium is obtained from the equations of state for the distortion tensor. It is shown that the sound is described as "massive" wave of the distortion tensor, the spectrum of which has the minimal frequency, which corresponds to a gap. The presence of a gap in the low-symmetric state gives reason to believe that the distortion tensor, as the compensating interaction field, describes a strong fundamental interaction. As it is known, the description of the gap in the strong fundamental interaction has been declared a Millennium problem by the Clay Mathematical Institute.
\end{abstract}

\section{Hosted file}

Braginsky_05_gap_21.pdf available at https://authorea.com/users/461652/articles/557287-theminimal-interaction-induced-by-the-translation-subgroup-has-a-gap-in-the-low-symmetricstate 